\title{
Influência da fadiga no equilíbrio do pé de apoio de jogadores de futebol
}

CDD. 20.ed. 796.023

796.33

\author{
Wakson Batista de Morais GOMES* \\ João BARTHOLOMEU NETO*/** \\ Claudio Oliveira ASSUMPÇÃO ${ }^{* * *}$ \\ Carina Helena Wasem FRAGA ${ }^{* * * * *}$ \\ Roberto BIANCO ${ }^{* * * * *}$ \\ Lais TONELLO** \\ Marcelo Magalhães SALES ${ }^{* *}$ \\ Ricardo Yukio ASANO ${ }^{* * *}$
}

${ }^{*}$ Centro Universitário UNIRG.

** Universidade Católica de Brasília.

*** Universidade Estadual Paulista. **** Escola de Educação Física e Esporte, Universidade de São Paulo.

\section{Resumo}

0 objetivo do presente estudo foi investigar a influencia da fadiga no equilíbrio do pé de apoio no momento do chute em jogadores de futebol. Participaram desta pesquisa 19 atletas de futebol divididos em dois grupos: grupo exaustão (GEX) que realizou um esforço com carga incremental até a exaustão em esteira rolante, e o grupo controle (GCE) realizou um esforço leve-constante de forma continua em esteira rolante. Antes e após os protocolos foram avaliados parâmetros de equilíbrio estático em apoio unipodal, utilizando uma plataforma de força. 0 GEX apresentou aumento da área de deslocamento do centro de pressão (COP) $(p<0,05)$ após exaustão, tanto para o pé direito como para o esquerdo. $A$ velocidade máxima de deslocamento do centro de pressão ântero-posterior da perna direita do GEX aumentou significantemente $(p<0,05)$ após a exaustão em relação aos valores de repouso. A fadiga pode diminuir a capacidade de equilíbrio do pé de apoio em jogadores de futebol. Principalmente no apoio com o pé que não é o usual de apoio para o chute.

Palavras-chave: Fadiga; Equilibrio; Futebol.

\section{Introdução}

A qualidade de execução dos fundamentos de uma modalidade esportiva como futebol depende de habilidade específica do esporte e de capacidades físicas como o equilíbrio corporal (EC). A cada nova postura corporal adquirida durante o jogo, respostas neuromusculares são necessárias para manter o equilíbrio ${ }^{1}$ para, consequentemente, executar as ações do jogo com maior eficiência possível.

O EC depende da estabilidade do Centro de Massa (CM) que pode ser influenciado pelo tamanho da base de apoio, pela projeção do CM nessa base de apoio, pela altura do $\mathrm{CM}$ e, ainda, pela massa corporal de cada indivíduo ${ }^{2}$. No intuito de avaliar as oscilaçōes em torno da base apoio, plataformas de força são freqüentemente utilizadas ${ }^{3}$. Para tanto, dados de Força de Reação do Solo, são utilizados para cálculo do Centro de Pressão (COP), que representa uma medida de deslocamento resultante das forças aplicadas no apoio. O COP é uma variável geralmente associada aos estudos do controle postural devido a sua relação com o centro de massa do corpo ${ }^{4}$.

Estudos têm sido realizados com objetivo de melhor entender a relação entre equilíbrio e o desempenho no futebol, considerando aspectos como a incidência de lesões no aparelho locomotor $^{5}$, a eficiência no treinamento proprioceptivo ${ }^{6}$, o desenvolvimento de $\mathrm{EC}$ em crianças $^{7}$, a prevenção de quedas evitando lesōes ${ }^{8}$, dentre outros. Porém a produção ainda é insuficiente para elucidar as questōes pertinentes ao equilíbrio em jogadores de futebol, principalmente relacionado a influencia da fadiga no equilíbrio desses atletas. 
O futebol é um esporte caracterizado por esforços intermitentes de alta intensidade, e que exige bom desenvolvimento de valências físicas como força, velocidade, e resistência9 ${ }^{9}$. Dessa forma, durante uma partida de futebol, tais açôes motoras podem desencadear um processo de fadiga muscular.

A fadiga muscular representa um fenômeno complexo que engloba várias causas, mecanismos e formas de manifestações, e se caracteriza por uma gradual redução na capacidade de produção de força ${ }^{10-11}$.

\section{Método}

\section{Amostra}

$\mathrm{O}$ projeto de pesquisa foi submetido e aprovado pelo Comitê de Ética em Pesquisa do Centro Universitário UNIRG (parecer 0183/2010) de acordo com as diretrizes estabelecidas na Resolução 196/96 do Conselho Nacional de Saúde.

Os voluntários da pesquisa assinaram um Termo de Consentimento Livre e Esclarecido, concordando sua participação nos procedimentos experimentais. Participaram do estudo 19 atletas de futebol de um mesmo clube profissional que competem em nível estadual e nacional no estado de Tocantins, com idade média de 16,5 $\pm 1,7$ anos, índice de massa corporal de $21,1 \pm 3,3 \mathrm{~kg} \cdot \mathrm{m}^{2}$. Os sujeitos foram submetidos a exames clínicos e anamnese ortopédica para assegurar a integridade ósteo-mio-articular do aparelho locomotor na época da coleta. Todos os atletas eram de dominância destra nos membros inferiores.

\section{Procedimentos}

Para investigar a influencia da fadiga sobre o equilíbrio do pé de apoio de jogadores de futebol, a amostra foi dividida aleatoriamente em dois grupos: grupo exaustão $(\mathrm{GEX})(\mathrm{n}=10)$ e grupo controle esteira (GCE) $(\mathrm{n}=9)$. Ambos os grupos foram submetidos a avaliaçôes de equilíbrio com apenas um apoio (um pé), realizadas em plataforma de força (AMTI OR6-5), pré e pós 12 minutos de corrida na esteira rolante. O grupo GEX realizou um teste incremental de esforço realizada até a exaustão voluntária (protocolo de Bruce), enquanto que o grupo GCE realizou o mesmo protocolo que o grupo GEX, porém com velocidade e inclinação constante referente à primeira fase do protocolo de Bruce (esse grupo, apesar de realizar esforço físico,
A influência da fadiga no equilíbrio do pé de apoio do chute ainda é pouco estudado, e consequentemente são inconclusivos. Portanto, o presente estudo tem como objetivo investigar a influencia da fadiga no equilíbrio do pé de apoio do chute em jogadores de futebol. A hipótese do estudo é que a fadiga pode diminuir a capacidade de controle neuromuscular influenciando negativamente o equilíbrio estático do pé de apoio, sendo essa mecânica importante para a precisão e qualidade das habilidades de chute e passe no futebol.

foi considerado controle, pois o intuito era de eliminar a variável de confundimento relacionada a influencia da esteira rolante no desequilíbrio na plataforma de força).

A plataforma de força mediu as três componentes da Força de Reação do Solo: a componente vertical $(\mathrm{Fz})$, a componente ântero-posterior (Fy) e a componente médio-lateral $(\mathrm{Fx})$, determinando a velocidade máxima de deslocamento, desvio padrão e área do centro de pressão (COP).

A coleta de dados iniciou com o voluntário posicionado descalço no centro da plataforma de força para realizar o teste de equilíbrio. O teste consistia em permanecer por $30 \mathrm{~s}$ em duas condiçóes diferentes: apoio unipodal direito e unipodal esquerdo ${ }^{12}$. A ordem das condições foi realizada aleatoriamente. $\mathrm{O}$ voluntário foi instruído a olhar fixamente em um ponto posicionado na altura de seus olhos em uma parede a $2 \mathrm{~m}$ de distancia e permanecer imóvel durante a execução do teste.

Posteriormente à coleta na plataforma de força, os voluntários realizaram testes na esteira rolante. O GEX realizou um teste incremental máximo até a exaustão voluntária seguindo o protocolo de Bru$\mathrm{ce}^{13}$. O teste iniciou com uma marcha a $2.74 \mathrm{~km} \cdot \mathrm{h}^{-1}$ com inclinação de $10 \%$ por três minutos. Após esse primeiro estágio, houve o aumento na velocidade na esteira, seguindo uma tabela referencial, e o incremento de $2 \%$ a cada três minutos até que a exaustão voluntária do atleta fosse alcançada. Já o GCE realizou um teste modificado, no qual o primeiro estágio do protocolo, $2.74 \mathrm{~km} \cdot \mathrm{h}^{-1}$ com inclinação de $10 \%$, foi mantido por 12 minutos. A escolha desta duração se deu em função do tempo médio do teste de exaustão comumente realizado no laboratório.

Imediatamente após o término do teste incremental, os voluntários foram novamente 
posicionados sobre a plataforma de força para novo registro da velocidade máxima de deslocamento, desvio padrão e área do COP.

\section{Variáveis de análise}

Os parâmetros analisados foram extraídos do deslocamento do COP durante os testes de equilíbrio. Os parâmetros foram: a velocidade máxima do COP, que é a maior velocidade de deslocamento do COP obtido durante as oscilações que o mesmo sofre durante os ajustes posturais para a manutenção do equilíbrio nas condições do teste; área total de deslocamento do COP, que é o contorno externo da trajetória de deslocamento do COP, que consisti na área de deslocamento do centro de pressão de refe- rencia (primeiro centro de pressão determinado) para os centros de pressão coletados durante ao longo do teste, o calculo do COP foi realizado pelo "software" específico da plataforma de força; e o desvio padrão de deslocamento do COP durante o teste.

\section{Análise estatística}

As médias e desvios padrão dos parâmetros analisados permitiram a comparação intra e inter grupos. A normalidade de distribuição de dados foi confirmada com teste de Shapiro-Wilk, sendo que, posteriormente, os dados foram comparados utilizando uma ANOVA one way com "post hoc" de Tukey, com intervalo de confiança de 95\% (p $<0,05)$.

\section{Resultados}

$\mathrm{O}$ grupo GEX apresentou média de $\mathrm{VO}_{2 \text { pico }} \mathrm{de}$ $68,14 \pm 3,9 \mathrm{ml} . \mathrm{kg} \cdot \mathrm{min}$. Lembramos que o grupo GCE não realizou o teste até o final pois foi um grupo controle sem indução a fadiga, assim não obtemos o $\mathrm{VO}_{\text {2pico }}$ desse grupo.

A TABELA 1 apresenta a média e desvio padrão da velocidade máxima (Vmax) de deslocamento do desequilíbrio dos grupos GEX e GCE. A Vmax ânteroposterior (y) da perna direita do GEX obteve aumento significante $(\mathrm{p}<0,05)$ após a sessão de exaustão na esteira

rolante em relação aos valores pré- exaustão do mesmo grupo e quando comparado ao pós do grupo GCE.

O desvio padrão do centro de pressão não apresentou diferença significativa inter e intra grupos como demonstrado na TABELA 2.

O GEX apresentou aumento de desequilíbrio em relação à área de deslocamento do centro de pressão $(\mathrm{p}<0,05)$ do momento pré- exaustão para o pós e para o momento pós do grupo GCE, tanto para o pé direito como para o esquerdo (TABELA 3).

TABELA 1 - Média e desvio padrão da velocidade máxima de deslocamento (m/s) de jogadores de futebol após exaustão (GEX) e após sessão controle na esteira (GCE).

\begin{tabular}{|c|c|c|c|c|c|c|c|c|}
\hline & \multicolumn{2}{|c|}{$\begin{array}{c}\operatorname{Vmax} x \\
\text { (pé direito) }\end{array}$} & \multicolumn{2}{|c|}{$\begin{array}{c}\text { Vmax x } \\
\text { (pé esquerdo) }\end{array}$} & \multicolumn{2}{|c|}{$\begin{array}{c}\text { Vmax y } \\
\text { (pé direito) }\end{array}$} & \multicolumn{2}{|c|}{$\begin{array}{c}\text { Vmax y } \\
\text { (pé esquerdo) }\end{array}$} \\
\hline & Pré & Pós & Pré & Pós & Pré & Pós & Pré & Pós \\
\hline GEX & $10,9 \pm 6$ & $9,5 \pm 2$ & $7,7 \pm 1$ & $8,4 \pm 2$ & $10,6 \pm 3$ & $15,6 \pm 3^{*} \dagger$ & $11 \pm 3$ & $12 \pm 3$ \\
\hline GCE & $8,8 \pm 2$ & $9,1 \pm 2$ & $7,5 \pm 1$ & $9 \pm 3$ & $9,2 \pm 2$ & $10,1 \pm 3$ & $9,2 \pm 2$ & $7,7 \pm 1$ \\
\hline
\end{tabular}

GEX = grupo exaustão; $\mathrm{GCE}=$ grupo controle esteira;

Vmax $\mathrm{x}=$ velocidade máxima de deslocamento latero-lateral;

Vmax y = velocidade máxima de deslocamento Antero-posterior;

* $p<0,05$ em relação a Vmax y (pé direito) pré do mesmo grupo:

$t p<0,05$ em relação a Vmax y (pé direito) do grupo GCE.

TABELA 2 - Média e desvio padrão do desvio padrão $(\mathrm{cm})$ da oscilação postural de jogadores de futebol antes e após exaustão (GEX) e antes e após sessão controle na esteira (GCE).

\begin{tabular}{ccccccccc}
\hline & \multicolumn{2}{c}{$\begin{array}{c}\text { SD } \\
\text { (pé direito) }\end{array}$} & \multicolumn{2}{c}{$\begin{array}{c}\text { SD } \\
\text { (pé esquerdo) }\end{array}$} & \multicolumn{2}{c}{$\begin{array}{c}\text { SD } \\
\text { (pé direito) }\end{array}$} & \multicolumn{2}{c}{$\begin{array}{c}\text { SD y } \\
\text { (pé esquerdo) }\end{array}$} \\
\hline & Pré & Pós & Pré & Pós & Pré & Pós & Pré & Pós \\
GEX & $0,25 \pm 0,03$ & $0,27 \pm 0,06$ & $0,26 \pm 0,03$ & $0,28 \pm 0,06$ & $0,33 \pm 0,05$ & $0,38 \pm 0,08$ & $0,32 \pm 0,06$ & $0,38 \pm 0,09$ \\
GCE & $0,23 \pm 0,04$ & $0,23 \pm 0,04$ & $0,22 \pm 0,03$ & $0,22 \pm 0,06$ & $0,30 \pm 0,04$ & $0,33 \pm 0,07$ & $0,28 \pm 0,07$ & $0,32 \pm 0,01$ \\
\hline
\end{tabular}

GEX = grupo exaustão; $\mathrm{GCE}=$ grupo controle esteira;

SD $x=$ desvio padrão latero-lateral;

SD y = desvio padrão Antero-posterior. 
TABELA 3 - Média e desvio padrão da Área da oscilação postural $\left(\mathrm{mm}^{2}\right)$ de jogadores de futebol antes e após exaustão (GEX) e antes e após sessão controle na esteira (GCE).

GEX = grupo exaustão; GCE = grupo controle esteira;

${ }^{*} p<0,05$ em relação ao pré do mesmo grupo: tp $<0,05$ em relação ao pós do GCE.

\begin{tabular}{ccccc} 
& \multicolumn{2}{c}{ Área (pé direito) } & \multicolumn{2}{c}{ Área (pé esquerdo) } \\
\hline & Pré & Pós & Pré & Pós \\
GEX & $1,3 \pm 0,2$ & $2,0 \pm 0,5^{*} \dagger$ & $1,3 \pm 0,2$ & $2,0 \pm 0,1^{*} \dagger$ \\
GCE & $1,3 \pm 0,2$ & $1,3 \pm 0,3$ & $1,2 \pm 0,3$ & $1,4 \pm 0,6$ \\
\hline
\end{tabular}

\section{Discussão}

No teste incremental, o voluntário foi instruído e incentivado a realizar o esforço até a exaustão, além disso, o tempo médio da exaustão aproximou-se de 12 minutos, comprovando a condição de fadiga. Com isso, podemos afirmar que os resultados mostram que a fadiga influenciou o equilíbrio estático. Essa influencia pode ser observada na maior área de deslocamento do COP em ambos os apoios para o grupo GEX, pós-protocolo de exaustão, e a velocidade de deslocamento do COP, no eixo de y, para um dos apoios. Outro resultado interessante foi que na velocidade de deslocamento após a indução da fadiga, o pé direito obteve diferença significativa em relação à $V \max$ y (pé direito) pré do mesmo grupo, e em relação a Vmax y (pé direito) do grupo GCE, o que não ocorreu com o pé esquerdo que é comumente utilizado como pé de apoio em ações como chute e passe (todos os atletas da amostra eram destros). Isso pode ser um indicador da especificidade do gesto. Provavelmente, a perna direita, por estar na maioria das vezes envolvida com o gesto do chute, ela poucas vezes é solicitada para manter-se em equilíbrio estático unipodal durante uma partida. $\mathrm{O}$ que pode explicar porque a velocidade de deslocamento do COP do pé direito aumentou, após o teste de exaustão.

A fadiga em jogadores de futebol vem sendo estudada somente em valências como diminuição da potencia muscular, questôes táticas e técnicas ${ }^{14}$. As pesquisas relacionando a fadiga ao desequilíbrio em jogadores de futebol ainda são escassos.

A fadiga no futebol é um evento comum, e ocorre principalmente no segundo tempo da partida ${ }^{15}$. A fadiga muscular está relacionada com diminuição do desempenho em geral. SiLVA et al. ${ }^{16}$ observou aumento no tempo de reação em atividades esportivas após fadiga muscular. A fadiga também influencia parâmetros eletromiográficos e cinemáticos da corrida e do ciclismo em atletas ${ }^{17}$.

O futebol competitivo exige altas demandas fisiológicas para execução de suas ações motoras durante o jogo ${ }^{18}$ induzindo, comumente, os atletas a fadiga muscular. As causas da fadiga muscular com a prática de esporte podem estar relacionados a um aumento de amônia no sangue ${ }^{19}$, baixas concentraçōes de glicogênio muscular ${ }^{20-21}$, desidratação ${ }^{22}$ e diminuição do $\mathrm{pH}$ intramuscular ${ }^{23}$ podem diminuir a glicólise e disfunções na neurotransmissão muscular com acúmulo de potássio no músculo ${ }^{24}$.

Para a manutenção do equilíbrio corporal, o SNC deve ser informado continuamente sobre o CG, que são reconhecidos por receptores especializados, organizados em sistemas sensoriais específicos ${ }^{25-27}$. A fadiga pode diminuir a capacidade de propriocepção a partir de esforços extenuantes, resultando na diminuição de percepção da posição $\operatorname{articular}^{28}$. Sendo assim, esse procedimento pode ser um possível mecanismo para as alterações do equilíbrio em situaçôes de fadiga em atletas de futebol.

Corroborando com os resultados do presente estudo BARONI et al. ${ }^{29}$ realizaram um estudo com o objetivo de verificar o efeito da fadiga muscular induzida pelo exercício de alta intensidade sobre a estabilidade postural dinâmica de atletas de futebol durante o passe sobre uma plataforma estabilometrica antes e após a realização de um protocolo de exercício de intensidade máxima em cicloergômetro. Após o protocolo de fadiga, os atletas apresentaram aumento de $31 \%$ na velocidade média de deslocamento do COP. Os autores concluíram que a fadiga muscular é capaz de promover decréscimo da estabilidade postural de jogadores de futebol durante $o$ gesto motor do passe.

No futebol moderno, é imprescindível ao atleta dominar a técnica e saber chutar com as ambas as pernas, pois desta maneira não precisará perder fraçôes de tempo precioso para direcionar seu corpo em relação a bola, quando a mesma está em seu membro não-dominante. Os resultados deste estudo apontam para diferenças na manutenção do equilíbrio estático 
entre o pé esquerdo e o pé direito. Acredita-se que com um treinamento adequado a cada faixa etária é possível que o membro não dominante tenha desempenho similar ao membro dominante ${ }^{30}$.

Durante um passe ou uma finalização, o posicionamento do pé de apoio é um aspecto importante a se considerar, pois influencia a precisão, assim como velocidade e alcance da bola ${ }^{31}$. Além do posicionamento do pé de apoio, outras variáveis são importantes para a execução do chute como o equilíbrio do corpo, posição do PE de toque e força imposta a bola ${ }^{32}$.

$\mathrm{O}$ pé direito dos jogadores da amostra apresentou um indicador de que o equilíbrio foi afetado. Contudo, o mesmo não aconteceu com o pé esquerdo que é pé de apoio em ações como passe e chute (todos os voluntários da amostra usam preferencialmente o pé esquerdo como de apoio). Uma hipótese para essa explicação pode ser que o pé de apoio possua maior equilíbrio em função de exercer essa função com maior frequência em treinamentos e jogos como apoio para o chute do outro pé.

Vale a pena lembrar algumas limitações que a técnica usada neste estudo pode apresentar. Em primeiro lugar, o uso de uma avaliação estática para a analise do equilíbrio é um fator limitante, pois embora o pé de apoio esteja estático no instante do chute, o mesmo geralmente é precedido por um deslocamento no qual uma posição estática de duração muito curta é realizada para o chute. Essa característica torna a técnica usada para a análise do equilíbrio de baixa especificidade a este gesto esportivo. Uma outra limitação é a impossibilidade em determinar em que extensão o aumento na área de deslocamento do COP pode efetivamente comprometer a eficiência do chute. Por último, vale a pena lembrar a falta de utilização de eletromiografia das musculaturas envolvidas, para determinar a atividade dos músculos envolvidos bem como quais músculos estariam ativos na indução a fadiga, também entendemos como uma limitação do estudo. Além disso, sugerimos futuramente estudos sobre fadiga e equilíbrio dinâmico em jogadores de futebol, aproximando mais dos movimentos realizados durante o jogo.

Concluímos que a fadiga imposta afetou o equilíbrio estático do pé de apoio em jogadores de futebol. Esse equilíbrio pode exercer alguma influencia na capacidade de realização do chute, mas com os dados apresentados neste estudo, não há como saber em que extensão o chute pode ser afetado. Sugere-se em futuros estudos a análise do próprio gesto do chute com talvez outra variável independente como a precisão ou a potencia do chute.

\begin{abstract}
Influence of fatigue on balance of foot support of football players

The aim of this study was to investigate the influence of fatigue on the balance of the support leg kick in soccer players. 19 soccer players were divided randomly into two groups: group exhauston (GEX), underwent an effort with incremental load at exhaustion on a treadmill, and the control group (GCE) underwent effort with constant lower load. Parameters of static balance in single support (one foot) were evaluated before and after the protocols, using a force platform (AMTI OR6-5). The GEX showed enlargement of the displacement of center of pressure (COP) $(p<0.05)$ after exhaustion, for the right foot. The maximum displacement of the antero posterior center of pressure of the right leg of the GEX incresead significantly $(p<0.05)$ after exhaustion in relation to resting values. In conclusion, fatigue can impair the ability to keep balance for the support leg in soccer players. Mainly on support to the foot that is not the usual support for the kick.
\end{abstract}

KEY WORDS: Fatigue; Balance; Football. 


\section{Referências}

1. Duarte M, Freitas SMF. Revisão sobre posturografia. Rev Bras Fisioter. 2010;14:183-92.

2. Hall S. Biomecânica básica. Rio de Janeiro: Guanabara Koogan; 1993.

3. Amadio AC, Duarte M. Fundamentos biomecânicos para análise do movimento. São Paulo: Escola de Educação Física, Universidade de São Paulo; 1996.

4. Mochizuki L, Amadio AC. Aspectos biomecânicos da postura ereta: a relação entre o centro de massa e o centro de pressão. Rev Port Cienc Desp. 2003;3:77-83.

5. Alonso AC, Greve JMD, Camanho GL. Evaluating the center of gravity of dislocations in soccer players with and without reconstruction of the anterior cruciate ligament using a balance platform. Clinics. 2009;64:163-70.

6. Baldaço FO, Cadó VP, Souza J, Mota CB, Lemos JC. Análise do treinamento proprioceptivo no equilíbrio de atletas de futsal feminino. Fisioter Mov. 2010;23:183-92.

7. Lemos LFC, Alves RF, Teixeira CS, Mota CB. Futebol de campo e o equilíbrio corporal de crianças. Rev Bras Ciênc Mov. 2008;16. Disponível em: http://portalrevistas.ucb.br/index.php/RBCM/article/view/919/916.

8. Söderman K, Werner S, Pietilä T, Engström B, Alfredson H. Balance board training: prevention of traumatic injuries of the lower extremities in female soccer palyers? Knee Surg Sports Traumatol Arthrosc. 2000;8:356-63.

9. Izquierdo M, Ibañez J, Calbet JA, et al. Neuromuscular fatigue after resistance training. Int J Sports Med. 2009;30:614-23.

10. Vøllestad NK. Measurement of human muscle fatigue. J Neurosci Methods. 1997;74:219-27.

11. Cifrek M, Medved V, Tonković S, Ostojić S. Surface EMG based muscle fatigue evaluation in biomechanics. Clin Biomech (Bristol, Avon) 2009;24:327-40.

12. Harris GF, Riedel SA, Matesi DV, Smith PA. Signal stationarity in postural stability assessment of children. IEEE Eng Med Biol. 1992;11:57-8.

13. Araújo CGS. Teste de exercício: terminologia e algumas considerações sobre passado, presente e futuro baseado em evidências. Rev Bras Med Esporte. 2000;6:77-84.

14. Weineck J. Futebol total: o treinamento físico no futebol. São Paulo: Phorte; 2000.

15. Campos AN. Estudo da fadiga muscular no futebol: respostas crônicas e agudas [tese]. Porto: Uiversidade do Porto; 1999.

16. Silva BARS, Martinez FG, Pacheco AMP. Efeitos da fadiga muscular induzida por exercícios no tempo de reação muscular dos fibulares em indivíduos sadios. Rev Bras Med Esporte. 2006;12:85-9.

17. Silva SRD, Fraga CHW, Gonçalves M. Efeito da fadiga muscular na biomecânica da corrida: uma revisão. Motriz. 2007;13:225-35.

18. Campeiz JM, Oliveira PR. Análise comparativa de variáveis antropométricas e anaeróbias de futebolistas profissionais, juniores e juvenis. Mov Percepc. 2006;6:58-84.

19. Wilmore JH, Costill DL. Fisiologia do esporte e do exercício. Barueri: Manole; 2001.

20. Reilly T. Aspectos fisiológicos del fútbol. Cordoba: PubliCE; 2003.

21. Maughan RJ, Burke LM. Nutrição esportiva. Porto Alegre: Artmed; 2004.

22. Aragón-Vargas LF, Moncada-Jiménez J, Hernández-Elizondo J, Barrenechea A, Monge-Alvarado M. Evaluation of pre-game hydration status, heat stress, and fluid balance during professional soccer competition in the heat. European J Sport Sci. 2009;9:269-76.

23. Rossi L, Tirapegui J. Aspectos atuais sobre exercício físico, fadiga e nutrição. Rev Paul Educ Fís. 1999;13:67-82.

24. Rienzi E, Drust B, Reilly T, Carter JEL, Martin A. Investigation of anthropometric and work-rate profiles of elite South American International soccer players. J Sports Med Phys Fitness. 2000;40:162-9.

25. Morasso PG, Schieppati M. Can muscle stiffness alone stabilize upright standing? J Physiol. 1999;82:1622-6.

26. Stoffregen TA, Pagulayan RJ, Bardy BG, et al. Modulating postural control to facilitate visual performance Hum Mov Sci. 2000;19:203-20.

27. Schubert MC, Minor LB. Vestibulo-ocular physiology underlying vestibular hypofunction. Phys Ther. 2004;84:373-85.

28. Ribeiro F, Oliveira J. Efeito da fadiga muscular local na propriocepção do joelho. Fisioter Mov. 2008;21:71-83.

29. Baroni BM, Wiest MJ, Generosi RA, Vaz MA, Leal Junior ECP. Efeito da fadiga muscular sobre o controle postural durante o movimento do passe em atletas de futebol. Rev Bras Cineantropom Desempenho Hum. 2011;13:348-53.

30. Cobalchini R, Silva ER. Treinabilidade do membro inferior não-dominante em atletas infantis de futebol. EFDeportes. 2008;13. Disponível em: http://www.efdeportes.com/efd125/treinabilidade-do-membro-inferior-nao-dominante-ematletas-infantis-de-futebol.htm.

80 • Rev Bras Educ Fís Esporte, (São Paulo) 2013 Jan-Mar;27(1):75-81 
31. Melo S, Lara Junior AA. Interferência do ângulo do pé de apoio no ângulo de saída da bola no chute no futebol com bola parada. XII Congresso Brasileiro de Biomecânica; 2007; Rio Claro, BR. Rio Claro: UNESP; 2007.

32. Melo RS. Trabalhos técnicos para o futebol. Rio de Janeiro: Sprint; 1999.

\begin{tabular}{r|r} 
ENDEREÇo & \\
Wakson Batista de Morais Gomes & Recebido para publicação: 08/05/2012 \\
Centro Universitário UNIRG & Aceito: 05/11/2012 \\
Av . Rio de Janeiro - Setor Central & \\
77403-090 - Gurupi - TO - BRASIL & \\
e-mail: waksontorinha@hotmail.com &
\end{tabular}

Rev Bras Educ Fís Esporte, (São Paulo) 2013 Jan-Mar;27(1):75-81 • 81 